\title{
Il ruolo della neurocriminologia nella costruzione di un profilo criminologico dell'omicida seriale*
}

\author{
Samuel Occhi, Manuela Martineæ, Luis Moya-Albiol ${ }^{\bullet}$
}

\section{Riassunto}

La neurocriminologia è una disciplina che si è sviluppata negli ultimi dieci anni. Il suo obiettivo principale è di studiare le variabili biologiche e neurologiche relazionate con la violenza e, di conseguenza, la criminalità. Il suo intervento ha come obiettivo quello di diagnosticare, prevenire e intervenire sulla violenza in generale e sul crimine, seguendo il modello bio-psico-sociale della criminalità.

L'obiettivo di quest'articolo è di analizzare la costruzione di un profilo criminale dell'omicida seriale in funzione delle caratteristiche neurocriminologiche. Nella parte introduttiva è analizzato in maniera descrittiva il fenomeno del serial killer. Tale analisi descrittiva prende in considerazione le classificazioni attualmente utilizzate, la criminogenesi e la criminodinamica. Nella seconda parte si passano in rassegna le variabili neurocriminologiche associate a tale profilo criminale. Tra queste, le principali sono la genetica ed il funzionamento cerebrale.

\section{Résumé}

La neurocriminologie est une discipline qui s'est développée dans les dix dernières années. Son but est d'étudier les variables biologiques et neurologiques liées à la violence et, par conséquent, à la criminalité. Son intervention vise à diagnostiquer, prévenir et agir sur la violence en général, notamment sur les crimes, suivant le modèle bio-psychosocial de la criminalité.

L 'objectif de cet article est d'analyser les modalités permettant à des enquêteurs de déterminer le profil d'un tueur en série en fonction de ses caractéristiques neurocriminologiques.

Le phénomène du tueur en série est décrit dans l'introduction.Cette analyse descriptive prend en compte les classifications qui sont actuellement utilisées, la criminogenèse et la criminodynamique. Dans la deuxième partie, les variables neurocriminologiques associées à ce profil criminel sont passées en revue. Parmi elles, la génétique et le fonctionnement cérébral sont les variables principales.

\section{Abstract}

Neurocriminology is a discipline which has developed over the last decade. Its aim is to study biological and neurological variables related to violence and, consequently, to criminality. Its intervention is intended to diagnose, prevent and work on violence in general, particularly on crime, following the bio-psycho-social model of criminality. The purpose of this article is to analyse modalities permitting the investigators to determine the profile of a serial killer according to their neurocriminological characteristics.

The phenomenon of serial killing is described in the introduction. This descriptive analysis takes into account the typically used classifications, the criminogenesis, and the criminodynamics. In the second part, neurocriminological variables associated with this criminal profile are reviewed. Among them, the main variables are genetics and brain functioning.

Key words: neurocriminology; violence; prevention; serial killer; profile.

\footnotetext{
* Gli autori ringraziano il professore di lingua italiana Denis Zamaro per l'assistenza linguistica e la correzione dell'articolo.

Questa ricerca fa parte degli studi del Master in Neurocriminologia dell'Università di València (Spagna) diretto dai professori Luis Moya-Albiol e Manuela Martínez.

- Departament de Psicobiologia, Universitat de València, Spagna.
} 


\section{Introduzione.}

Gli assassini seriali interessano maggiormente l'aspetto mediatico piuttosto che quello scientifico, come dimostra lo scarso numero di studi scientifici presenti. Tali soggetti presentano caratteristiche molto differenti tra loro, non soltanto per il numero, la tipologia di vittime coinvolte o per il movente che li muove, ma anche per le modalità esecutive con le quali vengono commessi gli omicidi ${ }^{1}$. Il primo caso di rilevanza mondiale di omicida seriale è quello di Jack lo Squartatore (Jack the Ripper) che, nella Londra di fine Ottocento, uccise sventrandole cinque giovani donne ${ }^{2}$. Negli ultimi vent'anni tale fenomeno ha interessato molte persone, anche grazie alla rilevanza data dai massmedia, includendo molte serie televisive e film. L'espressione "serial killer" viene coniata intorno agli anni '70 con il verificarsi, negli Stati Uniti, dei primi casi tra i quali quelli di Ted Bundy e di Juan Vallejo Corona ${ }^{3}$. Nella maggior parte degli eventi, si stima intorno al 90\%, l'omicida seriale è un giovane uomo che ha trascorso un'infanzia e/o un'adolescenza traumatica, spesso accompagnata da veri e propri abusi sessuali e/o fisici ${ }^{4}$. I casi più studiati si trovano negli Stati Uniti anche se, recentemente, si sono analizzati tale genere di fatti criminosi anche nel mondo

\footnotetext{
1 Gacono C. B., Hutton H. E., "Multiple murder: a review", Journal of British Criminology, Delinquency and Deviant Social Behavior, 34, 1, 1994, pp. 1-14.

${ }^{2}$ Wolf G., "A kidney from hell? A nephrological view of the Whitechapel murders in 1888", Nephrology Dialysis Transplantation, 23, 2008, pp. 3343-3349.

${ }^{3}$ Douglas J. E., Burgess A.W., Burgess A.G., Ressler R.K., Crime classification manual: A standard system for investigating and classifying violent crimes, Simon and Schuster, New York, 1992.

4 Holmes R.M., DeBerger J., Serial Murder, Sage, Newbury Park CA, 1988.
}

orientale $^{5}$. Sul fenomeno degli omicidi seriali italiani poche sono state le ricerche scientifiche effettuate. In Italia, le perizie psichiatriche che attestano la totale o parziale infermità mentale dei criminali si rifanno esclusivamente a diagnosi psicopatologiche. Non sono quindi prese in considerazione le caratteristiche genetiche o le alterazioni del circuito cerebrale che costituiscono elementi di ricerca della neurologia.

Segue un elenco dei principali assassini seriali italiani:

- Vincenzo Verzeni, 1867-1872, 2 vittime;

- Leonarda Cianciulli, 1939-1940, 3 vittime;

- “il mostro di Firenze", 1968-1985, 14-16 vittime;

- il caso "Ludwig" (Marco Furlan e Abel Wolfgang), 1977-1984, 28 vittime;

- Maurizio Minghella, 1978-2001, 15 vittime;

- Andrea Matteucci, 1980-1990, 4 vittime;

- Roberto Succo, 1981-1987, 7 vittime;

- Luigi Chiatti, 1993, 2 vittime;

- Gianfranco Stevenin, 1993-1994, 6 vittime;

- Milena Quaglini, 1995-1999, 3 vittime;

- Donato Bilancia, 1997-1998, 17 vittime.

L'argomento degli omicidi seriali ha come principale input teorico il concetto della violenza e quello di aggressività. In funzione dell'atto si differenzia la violenza in fisica, sessuale, psicologica. Questa classificazione è abbastanza obiettiva e le informazioni su ogni tipologia possono essere completate con la frequenza, l'intensità e la durata in cui viene eseguita. La

\footnotetext{
${ }^{5}$ Dogra T. D., Leenaars A. A., Chadha R. K., Manju M., Laiwani S., Sood M., Lester D., Raine E., Behera C., "A psychological profile of a serial killer: a case report”, Omega, 65, 2012, pp. 299-316.
} 
violenza inoltre può essere classificata in violenza rivolta a se stessi, violenza interpersonale e violenza collettiva. In funzione dell'età si classifica la violenza in infantile, giovanile e adulta e in funzione del genere, maschile o femminile. La violenza infine può essere impulsiva, anche denominata emozionale, reattiva, ostile o affettiva, premeditata, denominata anche strumentale o proattiva e violenza collegata ad alterazioni mediche ${ }^{6}$. L'aggressività è definita come "un comportamento che si traduce in danni alla persona o alla proprietà"7 o "l'intenzione di infliggere un danno ad altri"8. Distinguiamo l'aggressività strumentale, in altre parole il comportamento razionale e calcolato utilizzato da un individuo per massimizzare i suoi profitti che può riguardare i serial killer e gli psicopatici, dall'aggressività impulsiva in cui l'obiettivo è soltanto quello di infliggere danni o ferite alla vittima.

Il focus di quest'articolo è quello di analizzare i fattori che influiscono nella costruzione di un profilo criminologico dell'omicida seriale, considerando gli studi più recenti che si rifanno alla disciplina della neurocriminologia.

\section{Omicidi in serie: definizioni.}

La frequenza degli omicidi seriali negli Stati Uniti ha avuto un picco storico negli anni '80 del XX secolo e la maggior parte dei casi ha visto come protagonisti persone di razza bianca? ${ }^{9}$ Il

${ }^{6}$ Moya-Albiol L., Psicobiologia de la violencia, Piramide, Madrid, 2010.

7 Bandura A., Aggression: A social learning analysis, Prentice-Hall, Englewood Cliffs, NJ, 1973.

${ }^{8}$ Baron R. A., Byrne D., Social psychology (10th edition), Allyn \& Bacon, Boston, 2003.

${ }^{9}$ Canter D. V., Alison L. J., Alison E., Wentink N.,

"The organized/disorganized typology of serial fenomeno italiano segue il trend europeo ed è assai ridotto rispetto a quello statunitense $\mathrm{e}^{10}$.

Una delle classificazioni più diffuse è quella proposta dalla Federal Bureau of Investigation che suddivide gli omicidi seriali in tre categorie ${ }^{11}$. Il mass murder o omicida di massa è colui che uccide almeno quattro vittime in un medesimo luogo e durante uno stesso evento ${ }^{12}$. Il soggetto non conoscerebbe le sue vittime, che per lo più sarebbero scelte casualmente ${ }^{13}$. Lo spree murder o assassino compulsivo uccide due o più vittime, in luoghi diversi ma adiacenti, in un lasso di tempo molto breve. Il soggetto agirebbe lasciando molte tracce e di conseguenza tende ad essere catturato con facilità. Solitamente non conosce le vittime e, dato che non nasconde le sue tracce, continua a uccidere finché non viene catturato. Il serial murder o serial killer propriamente detto è un soggetto che uccide almeno tre vittime, in eventi distinti, in luoghi separati e con un intervallo di tempo fra un omicidio e l'altro. Può colpire a caso o, al contrario, scegliere accuratamente le sue vittime. Spesso ritiene di essere invincibile e che non verrà mai catturato, per questo, sovente, ama sfidare le forze dell'ordine ${ }^{14}$. Sceglierebbe spesso le vittime sconosciute più vulnerabili come bambini, adolescenti, donne

murder: Myth or model?”, Psychology, Public Policy and Law, 10, 2004, pp. 293-320.

10 Harbort S,. Mokros M., "Serial Murderers in Germany from 1945 to 1995. A descriptive study", Homicide Studies, vol. 5, No. 4, 2001, pp. 311-334.

11 Petherick W.A., Serial crime: Theoretical and Practical issues in behavioral profiling, Elsevier, California, 2006.

12 Holmes R., Holmes S., "Understanding mass murder: a starting point", Federal Probation, 49, 1992, pp. 29-34.

13 Rappaport R., "The serial and muss murder: patterns, differentiation, pathology", American Journal of Forensic Psychiatry, 9, 1988, pp. 39-48.

14 Turvey B., Criminal profiling. An introduction to behavioral evidence analysis, Elservier, California, 2008. 
(molte delle quali prostitute) ${ }^{15}$. Per l'assassino la vittima spesso può avere un valore simbolico ${ }^{16}$, motivo per cui il metodo di uccisione può variare $^{17}$. Recentemente è stata presa in considerazione una quarta categoria che corrisponde al serial-mass murder, tutt'ora in fase di studio e classificazione ${ }^{18}$.

Altre tipologie di assassini seriali sono: il ritual serial killer, che uccide per motivazioni ritualistiche; l'arsonist serial killer, anche conosciuto come Unabomber dopo il caso di Theodore John Kaczynski; lo sniper serial killer o omicida seriale cecchino, che uccide in maniera indiscriminata e senza una logica temporale con modalità tipiche dei cecchini. Infine, il serial killer by proxy è rappresentato dal caso del dottor Sigvard Thurneman, uno psichiatra svedese specializzato in ipnosi che ha utilizzato la sua capacità di influenzare il prossimo per indurre alcuni pazienti a commettere una serie di crimini, tra cui rapine ed omicidi.

Attualmente, quindi, si è concordi nello stabilire che il profilo del serial killer medio è un uomo bianco, di status socio-economico medio-basso, tra i 20 ed i 35 anni, anche se si sono osservati casi di assassini aventi un'età inferiore ai 20 anni ${ }^{19}$.

15 Kraemer G.W., Lord W.D., Heilburn K., "Comparing single and serial homicide offenses", Behavioral Science of Law, 22, 2004, pp. 325-343.

16 Rice M.E., "Violent offender research and implications for the criminal justice system", American Psychologist, 52, 1997, pp. 414-423.

17 Newton M., The Encyclopedia of serial killers, 2 ed., Checkmark Books, New York, 2006.

18 Edelstein A., "Re-thinking typologies of multiple murders: the missing category of serial-mass murder and its theoretical and practical implications", Journal of International Mental Health, 16, 2014, pp. 350-353.

19 Myers W.C., "Serial murder by children and adolescents", Behavioral Science of Law, 22, 2004, pp. 357-374.

\section{Criminodinamica dell'omicidio seriale.}

Importanti informazioni circa l'analisi e la classificazione degli omicidi seriali emergono sia dall'indagine del movente ${ }^{20}$ e dal numero delle vittime, sia dalle dinamiche tramite le quali essi vengono commessi ${ }^{21}$. La classificazione operata dalla Federal Berau of Investigation li distingue in organizzati e disorganizzati22 (vedasi tabella n. 1). L'omicida seriale organizzato è colui che pianifica con meticolosità i propri delitti selezionando le vittime. Si ipotizza che possa trattarsi di soggetti dotati di un alto QI, capaci di interagire con il tessuto sociale e ben inseriti allinterno del contesto familiare. Compiono l'omicidio con premeditazione e meticolosità e mostrano un forte autocontrollo durante il crimine. Dopo l'eliminazione della vittima cercano di occultarne il cadavere e di lasciare poche tracce sul luogo del delitto. L'uccisione viene di solito compiuta lentamente, infierendo e torturando. Un altro elemento caratteristico è la propria "firma" sul luogo del delitto che consiste nel lasciare oggetti, simboli, biglietti, messaggi di altro genere da parte del killer.

L'omicida seriale definito disorganizzato non agisce con premeditazione e il delitto avviene sotto l'impulso del momento. Questa è la ragione per la quale egli lascia spesso numerose prove sulla scena del crimine compreso il cadavere che, spesso, viene abbandonato sul

\footnotetext{
${ }^{20}$ Sears D.J., To kill again: The motivationand development of serial murder, Scholarly Resources, Inc., Wilmington, DE, 1991.

${ }^{21}$ White J.H., Lester D., Gentile M., Rosenbleeth J., "The utilization of forensic science and criminal profiling for capturing serial killers", Journal of Forensic Science International, 209, 2011, pp. 160-165.

22 Douglas J. E., Burgess A.W., Burgess A.G., Ressler R.K., Crime classification manual: A standard system for investigating and classifying violent crimes, Simon and Schuster, New York, 1992.
} 
luogo del delitto. L'arma utilizzata è sovente impropria, trattasi ad esempio di spranghe, di oggetti contundenti, di pietre, a differenza dell'assassino seriale organizzato che utilizza prevalentemente lo strangolamento, coltelli o armi da fuoco. L'omicida seriale disorganizzato, quindi, agirebbe per un impulso improvviso che lo porta a uccidere vittime scelte casualmente, senza preoccuparsi di cancellare tutte le sue tracce, pertanto sarebbe molto più facile da catturare.

Hickey classifica gli uccisori seriali in itineranti, locali e stazionari. Gli assassini seriali itineranti o viaggiatori $(28 \%)$ sono soggetti che spesso coprono lunghe distanze, uccidendo vittime in diverse regioni o stati. Gli assassini seriali locali cercano vittime nella stessa regione o stato in cui hanno compiuto il primo omicidio, mentre quelli seriali stazionari (45\%) rimangono in una specifica zona geografica ${ }^{23}$.

De Luca e Mastronardi distinguono l'omicida seriale classico da quello atipico ${ }^{24}$. Il serial killer classico è colui che uccide le vittime utilizzando delle metodiche che gli permettono di avere un contatto fisico con le vittime. Asporta feticci o "trofei" dal cadavere, che ama conservare per rivivere gli omicidi nella sua mente e sperimentare un nuovo piacere anche dopo molto tempo. Nella categoria "atipica" rientra l'omicidio mafioso e terroristico seriale con caratteristiche di sadismo oppure quei soggetti che utilizzano metodiche singolari come per esempio armi peculiari.

Riguardo al numero di killer coinvolti nell'azione omicida si possono distinguere quattro categorie.

23 Hickey E. W., Serial murderers and theirs victims, Wadsworth Publishing Company, Belmont, 1997.
L'omicida seriale individuale, che agisce da solo e che rappresenta la tipologia più conosciuta. L'omicida seriale in coppia è caratterizzato dalla presenza di due individui che compiono insieme i delitti. Si parla di coppia assassina anche quando, in realtà, è uno solo dei soggetti a commettere concretamente l'omicidio, mentre l'altro assiste al fatto e aiuta poi a disfarsi del cadavere. Le coppie sono sempre formate da una persona con personalità dominante $\mathrm{e} \mathrm{da}$ una con personalità sottomessa. Il soggetto dominante, che nelle coppie uomo/donna è quasi sempre l'uomo, pianifica l'azione omicida e la metterebbe in atto anche senza la presenza dell'altro. L'altro membro della coppia è un soggetto passivo, che non è in grado di opporre resistenza alla volontà del dominante. Gli omicidi seriali di gruppo sono commessi da tre o più persone. Gli assassinii di questo tipo possono essere compiuti da tutti i membri del gruppo, oppure ci può essere un soggetto deputato a portare a termine l'azione omicida, mentre gli altri si rendono complici non facendo nulla, di fatto, per impedire le uccisioni.

Altre tipologie di serial killer sono l'omicida seriale per eutanasia che agisce con l'apparente motivazione di dover salvare persone che soffrono ingiustamente. I casi di omicidio seriale di questo genere sono piuttosto numerosi e vedono coinvolti soprattutto operatori sanitari. Gli omicidi sono commessi in modo da far pensare ad una morte naturale. È molto difficile scoprire questo tipo di delitto seriale in quanto $\mathrm{i}$ sintomi provati dalle vittime sono compatibili con un decesso per cause naturali. L'omicida seriale, per il fatto di essere al centro

${ }^{24}$ Mastronardi V., Manuale per operatori criminologici e 
dell'attenzione, crea volontariamente una situazione di pericolo per le vittime e, in seguito, tenta, inutilmente, di salvarle allo scopo di assumere un atteggiamento da eroe. Questo tipo di omicidio viene di solito attuato da donne che mettono in pericolo la vita dei propri figli o di altri bambini. L'omicida seriale sadico si distingue per il piacere che prova nell'uccidere e frequentemente uccide le vittime solo dopo averle torturate a lungo. In questo caso il piacere principale sta nell'infliggere alle vittime il massimo dolore fisico e psicologico. Nell'omicida seriale sessuale il genere di atto sessuale e il suo significato simbolico variano a seconda della personalità dell'assassino ${ }^{25}$ ed i serial killer sessuali vengono identificati perché nell'acting out delle proprie fantasie lasciano una firma caratteristica sul corpo delle vittime o sulla scena del crimine $^{26}$. La maggior parte degli omicidi seriali sessuali avrebbero un intenso interesse per voyeurismo, feticismo e porno-sadomaso in età molto precoce ${ }^{27}$. Si possono distinguere due sottogruppi: omicidio seriale sessuale sadico caratterizzato da gratificazione sessuale ottenuta, da parte del killer, infliggendo grandi sofferenze alle vittime, e omicidio seriale sessuale necrofilo, il cui autore uccide le vittime nel modo più veloce possibile e con una metodica non lesiva dei tessuti corporei. In questo caso, a distanza di alcuni giorni dall'omicidio, l'assassino può

psicopatologi forensi, Giuffrè, Milano, 2012.

25 Groth A. N., Burgess A.W., Holmstrom L.L., "Rape: Power, anger and sexuality", American Journal of Psychiatry, 134(11), 1977, pp. 1239-1243.

${ }^{26}$ Ressler R. K., Burgess A.W., Douglas J.E., Sexual Homicide: patterns and motives, Iosington, New York, 1998.

27 Warren J., Hazelwood R.R., Dietz P.E., "The sexually sadistic serial killer", Journal of Forensic Science, 41, 1996, pp. 970-974. decidere di sezionare il cadavere per conservarne alcune parti.

Una ulteriore classificazione suddivide gli omicidi seriali in base alla pianificazione. L'omicidio seriale a pianificazione zero viene compiuto seguendo un impulso momentaneo e senza organizzazione. L'omicidio seriale a pianificazione mista è un evento in cui l'assassino alterna l'organizzazione e la disorganizzazione. L'omicidio seriale a pianificazione fasica viene pianificato solo in alcune delle fasi esecutive del progetto, mentre altre avvengono d'istinto.

Abbiamo finora visto che la maggior parte degli autori degli omicidi seriali sono uomini, ad eccezione dell'omicida seriale per eutanasia. Tuttavia, non mancano casi compiuti da donne ${ }^{28}$. La classificazione forse più esaustiva è quella elaborata da Hickey che le distingue in due gruppi: le vedove nere e gli angeli della morte, ovvero infermiere o operatori sanitari che uccidono i pazienti. Le vedove nere comincerebbero l'attività criminale verso $\mathrm{i}$ venticinque anni. Le vittime spesso fanno parte dell'entourage familiare, incluso il proprio marito, compagno, familiari o persone con le quali esse hanno stabilito una relazione. Il metodo usuale è di fare ingerire un veleno, il che rende molto difficile l'incriminazione e la motivazione è sovente di tipo economico. Gli angeli della morte inizierebbero il loro percorso violento verso i ventuno anni e in un luogo ben definito quale un ospedale, una clinica privata ${ }^{29}$. In questo caso la motivazione non sarebbe economica, bensì una

\footnotetext{
${ }^{28}$ Wilson W., Hilton T., "Modus operandi of female serial killers", Psychology Rep., 82, 1998, pp. 495-498. ${ }^{29}$ Yorker B.C., Kizer K.W., Lampe P., Forrest A.R., Lannan J.M,. Russell D.A., "Serial muder by healthcare professionals", Journal of Protocol Manage, 24, 2008, pp. 63-77.
} 
sensazione di potere generata dal fatto che la vita o la morte di una persona è nelle loro mani. Solitamente utilizzano il cloruro di potassio che simula attacchi cardiaci. Un esempio è quello di Terry Rachals che uccise sei persone e fu condannata solamente per lesione aggravata ${ }^{30}$.

\section{Criminogenesi dell'omicidio seriale.}

I ricercatori stanno lavorando da molti anni per capire che cosa induce una persona a diventare un omicida seriale, ma non vi è ancora nessuna risposta univoca, anche se si sono definite alcune caratteristiche che li accomunano ${ }^{31}$. Uno dei principali fattori considerati come scatenante la condotta degli assassini seriali è l'aver sofferto un'infanzia particolarmente traumatica e/o un abuso sessuale infantile ${ }^{32}$. Abuso che può essere stato compiuto da uno sconosciuto o da un membro della famiglia, sul cui ricordo è intervenuta una rimozione cognitiva ${ }^{33}$. A riguardo, uno dei lavori più conosciuti a livello scientifico è quello di Dietz e collaboratori, i quali studiarono casi di assassini seriali tra il 1984 e il 1988. Tali ricercatori conclusero che la maggior parte di essi denunciava di essere stato vittima di abuso sessuale nell'infanzia ${ }^{34}$. Esistono altri tre fattori collegati alla genesi di un omicidio seriale: la piromania, la crudeltà verso animali e

${ }^{30}$ Ostrosky-Solis F., Velez-Garcia A., Santana-Vargas D., Perez M., Ardilla A., "A middle-aged female serial killer", Journal of Forensic Science, 53, 2008, pp. 12231230.

${ }^{31}$ Egger S.A., The killer among us: an examination of serial murder and its investigation, Prentice Hall, Upper Saddle River, NJ, 1998.

32 Sycamnias E., Evaluating a Psychological Profile of a Serial Killer, 2007. Da: http://www.crime.about.com

${ }^{33}$ Mitchell H., Aamodt M.G., "The incidence of child abuse in serial killers", Journal of Police and Criminal Psychology, 20, 2005, pp. 40-47.

34 Dietz P. E., "Mass, serial and sensational homicides", Bulletin of the New York Academic of Medicine, 62(5), 1986, pp. 477-491. l'incontinenza urinaria infantile ${ }^{35}$. Più del $60 \%$ degli assassini seriali avrebbe sofferto di enuresi notturna durante l'infanzia e quasi tutti avrebbero torturato sadicamente animali ${ }^{36}$. Arnold Arluke e collaboratori hanno analizzato 153 soggetti che torturavano animali e li hanno paragonati ad un gruppo di controllo. Chi torturava animali aveva una probabilità cinque volte superiore di commettere atti di violenza come aggressione, stupro o omicidio. L'uccisione di animali consentirebbe alla persona di esercitare un dominio e un potere differentemente represso.

Oltre il 90\% degli assassini seriali tendono a provenire $\mathrm{da}$ famiglie molto instabili $\mathrm{O}$ disfunzionali, di solito abbandonati da uno dei due genitori, soprattutto dalla madre ${ }^{37}$. Vivere in una famiglia violenta può generare nei bambini un vissuto di costante paura che li indurrebbe a reprimere i sentimenti, ostacolandone la loro capacità di provare empatia ${ }^{38}$. In uno studio di 62 serial killer di sesso maschile, si è osservato che il $48 \%$ di loro era stato respinto da un genitore o da un'altra persona importante della loro vita. 亡̀ stato osservato che un gruppo di bambini con un genitore in carcere e/o ritenuto socialmente pericoloso presentava livelli elevati di delinquenza e di aggressività maggiore ${ }^{39}$.

35 Dogra T. D., Leenaars A. A., Chadha R. K., Manju M., Laiwani S., Sood M., Lester D., Raine E., Behera C., "A psychological profile of a serial killer: a case report", Omega, 65, 2012, pp. 299-316.

${ }^{36}$ Fisher K., Fisher R., Common characteristics of a serial killer, 2003. Retrieved August 8, 2014 from http://www.carpenoctem.tv

37 Salfati G., Canter D., "Differentiating Stranger Murders: Profiling Offender Characteristics from Behavioral Styles", Behavioral Sciences and the Law, 17, 1999, pp. 391-406.

38 Moya-Albiol L., La empatía. Entenderla para entender a los demás, Plataforma, Barcelona, 2014.

39 Loeber R., Pardini D., Homish L., Wei E. H., Crawford A. M., Farrington D.P., "The prediction of 
Altri fattori che incidono sullo sviluppo di una predisposizione caratteriale nei confronti della violenza nel bambino sono: comportamenti violenti in ambito familiare, abuso di sostanze, frequentazione di coetanei aggressivi, tendenza a reprimere la rabbia e un basso grado di resilienza ${ }^{40}$.

\section{Il criminale psicopatico.}

Nell'elaborazione di un concetto criminologico della psicopatia si ricorre a diverse discipline. La psichiatria e la psicologia considerano la psicopatia come un disturbo di personalità. La sociologia definisce lo psicopatico come un soggetto non in grado di relazionarsi socialmente con incapacità di assumere un atteggiamento empatico nei confronti degli altri. La biologia sottolinea il ruolo del sistema nervoso e la giurisprudenza considera la psicopatia come un disturbo mentale. Per la criminologia lo psicopatico sarebbe un individuo che soffre di un disturbo di personalità associato ad uno stile di vita socialmente inopportuno, con una tendenza ad ignorare le norme sociali.

La psicopatia è quindi considerata un disturbo mentale, nonostante sia poco considerata dai manuali diagnostici. Il suo spettro psicopatologico va da un deficit dell'empatia a comportamenti aggressivi con tendenze criminali. Il DSM classifica la psicopatia come un "pattern pervasivo d'inosservanza e di violazione dei diritti degli altri”, pattern anche denominato psicopatia, sociopatia o disturbo dissociale di

violence and homicide in young men", Journal of Counseling and Clinical Psychology, 73, 2005, pp. 10741088.

${ }^{40}$ Gao Y., Raine A., Venables P. H., Dawson M. E., Mednick S. A., "Association of poor childhood fear personalità ${ }^{41}$. Il disturbo antisociale della personalità si caratterizza per un totale disprezzo delle leggi e delle norme sociali. Spesso la persona con tale diagnosi presenta una lunga storia di arresti e di scontri fisici ${ }^{42}$.

La ricerca sui fattori di rischio nel disturbo antisociale di personalità si è focalizzata sull'individuazione di persone a rischio di commettere reati violenti ${ }^{43}$. Questa convinzione ha portato ad un crescente interesse per lo studio dei bambini e degli adolescenti ritenuti a rischio, perché attuano comportamenti antisociali o perché hanno già commesso reatiti, al fine di prevenire eventualmente comportamenti criminali futuri ${ }^{45}$. I bambini con caratteristiche antisociali tenderebbero a mostrare una scarsa capacità verbale, una spiccata impulsività, un'emotività negativa, insuccesso scolastico, alti livelli di ADHD (Attention-Deficit/Hyperactivity Disorder), disturbo oppositivo provocatorio e disturbo della condotta ${ }^{46}$.

conditioning and adult crime", American Journal of Psychiatry, 167, 2010, pp. 56-60.

${ }^{41}$ American Psychiatric Association, DSM-V, 2014.

42 Alison L., Bennell C., Mokros A., Ormerod, D., "The personality paradox in offender profiling: A theoretical review of the processes involved in deriving background characteristics from crime scene actions", Psychology, Public Policy and Law, 8, 2002, pp. 115-135.

${ }^{43}$ Herve H.F., Mitchell D., Cooper B.S., Spidel A., Hare R.D., "Psychopathy and unlawful confinement: An examination of perpetrator and event characteristics", Canadian Journal of Behavioural Science, 36, 2004, pp. 137-145.

44 Taylor J,. Iacono W.G., McGue M., "Evidence for a genetic etiology of early-onset delinquency", Journal of Abnormal Psychology, 109, 2000, pp. 634-643.

${ }^{45}$ Moffitt T.E., "The new look of behavioral genetics on developmental psychology: Gene-environment interplay in antisocial behaviors", Psychological Bullettin, 131, 2005, pp. 533-535.

46 Lahey B.B., Loeber R., Burke J.D., Applegate B, "Predicting future antisocial personality disorder in males from a clinical assessment in childhood", 
Eysenck operò una distinzione tra psicopatia primaria e psicopatia secondaria. La psicopatia primaria sarebbe quella maggiormente accompagnata da comportamenti omicidi e si caratterizzerebbe per assenza di sentimento di colpa, mancanza di empatia e di sensibilità nella persona che aumenterebbe la probabilità di commettere delitti ${ }^{47}$. Gli psicopatici criminali presenterebbero una mancanza totale di rimorso o di senso di colpa per le loro azioni48. L'arma utilizzata per compiere il crimine è, in genere, improvvisata e il passaggio all'atto, comunque disorganizzato, è assai violento. Alcuni assassini seriali possono essere identificati come soggetti affetti da psicopatia primaria, tra questi Gary Ridgeway, Ed Gein, Ted Bundy, Jeffrey Dahmer ${ }^{49}$, Dennis Rader, John Wayne Gacy e Ed Kemper. Diversa è la psicopatologia della psicopatia secondaria che potrebbe essere intesa come una difesa secondaria rispetto ad un altro tipo di psicopatologia predominante. La psicopatia melanconica, per esempio, corrisponde ad una difesa psicopatica secondaria e ad una melanconia di base. Lo stesso si verificherebbe nelle difese psicopatiche dei quadri ansiosi o fobici.

Gli studi che rievocano l'importanza dei fattori psicosociali nelle psicopatie enfatizzano problematiche nei legami familiari. Gao e collaboratori studiarono 333 famiglie di

Journal of Counseling and Clinical Psychology, 73, 2005, pp. 389-399.

47 Eysenck H.J., Eysenck S.B.G., Psychoticism as a dimension of personality, Hodder and Stoughton, London, 1976.

48 Montaldo C., Characteristics of a Psychopathic Personality, 2007. Retrieved January 10, 2015 from http://www.crime.about.com

${ }^{49}$ Silva J. A., Ferrari M.M., Leong G.B., "The case of Jeffrey Dahmer: Sexual serial homicide from a psicopatici e riscontrarono come elemento in comune una relazione genitoriale altamente conflittuale. Uno dei fattori che più si correlare con la psicopatia sarebbe quindi la presenza di maltrattamenti fisici o sessuali ${ }^{50}$ subiti all'interno dell'ambiente familiare ${ }^{51}$ All'esterno della famiglia, invece, esisterebbero diversi fattori in relazione con lo sviluppo di condotte antisociali, come ad esempio il rifiuto sociale e il bullismo. Sims fa notare che il criminale psicopatico, incapace di riconoscere la paura, agirebbe senza premeditazione e la vittima sarebbe frequentemente una persona conosciuta e/o appartenente all'entourage familiare.

Gli studi sulla relazione tra la psicopatia e i fattori biologici considerano soprattutto l'aspetto genetico. Una meta-analisi di Demmo e collaboratori evidenzia la presenza di un'alta relazione tra $\mathrm{i}$ fattori genetici e i tratti di personalità presenti in individui con psicopatia primaria. L'amigdala e la corteccia prefrontale della corteccia sono due delle aree del cervello che sono state maggiormente studiate nella psicopatia ${ }^{52}$. Il sistema limbico del cervello che contiene le strutture che controllano la memoria e le emozioni sembrerebbe altamente

neuropsychiatric developmental perspective", Journal of Forensic Sciences, 47, 2002, pp. 1-13.

${ }^{50}$ van Winkel R., van Nierop M., Myin-Germeys I., van Os J., "Childhood trauma as a cause of psychosis: linking genes, psychology and biology", Can J Psychiatry, 58, 2013, pp. 44-51.

51 Salekin R.T., Leistico A.M., Neumann C.S., DiCicco T.M., Duros R.L., "Psychopathy and comorbidity in a young offender sample: Taking a closer look at psychopathy's potential importance over distuptive behavior disorders", Journal of Abnormal Psychology, 113, 2004, pp. 416-427.

52 Yang Y., Raine A., Lencz T., Bihrle S., LaCasse L., Colletti P., "Volume reduction in prefrontal gray matter in unsuccessful criminal psychopaths", Biological Psychiatry, 57, 2005, pp. 1103-1108. 
compromesso nei criminali psicopatici ${ }^{53}$. Alcune lesioni della corteccia prefrontale, quindi, sarebbero legate alla psicopatia ${ }^{54}$. La corteccia orbito-frontale mediale riceve e invia proiezioni all'amigdala ed entrambe sarebbero deteriorate negli psicopatici55 56. Tali studi convergerebbero quindi verso un'eziologia biologica della psicopatia e di conseguenza dei comportamenti criminali ad essa associati ${ }^{57}$. Nella tabella n. 2 sono rappresentati in sintesi $\mathrm{i}$ tratti psicopatici e le regolazioni neurochimiche influenzate. Una classificazione utilizzata in Europa distingue tra criminale psicopatico e psicopatico senza condotte criminali. Questa classificazione nasce grazie agli studi di Robert Hare ${ }^{58}$ e alla relativa pubblicazione della Hare Psychopathy Checklist costituita dai seguenti criteri: 1) Loquacità; 2) Egocentrismo; 3) Tendenza alla noia e necessità costante di stimoli; 4) Menzogna patologica; 5) Manipolazione; 6) Mancanza di sentimento di colpa; 7) Scarsa profondità negli affetti; 8) Insensibilità e mancanza di empatia; 9) Stile di vita "parassita"; 10) Condotte sessuali promiscue; 11) Problematiche nella condotta in età precoce; 12) Assenza di obiettivi concreti; 13) Impulsività; 14) Irresponsabilità; 15) Relazioni

53 Kiehl K.A., Smith A.M., Hare R.D., "Limbic abmormalities in affective processing by criminal psychopaths as revealed by functional magnetic resonance imaging", Biological Psychiatry, 50, 2001, pp. 677-684.

54 Sycamnias E., Evaluating a Psychological Profile of a Serial Killer, 2007. Da: http://www.crime.about.com

55 Blair R. J., "Neurobiological basis for psychopathy", British Journal of Psychiatry, 182, 2003, pp. 5-7.

56 Tiihonen J., Hodggins S., Vaurio O., "Amygdaloid volume loss in psychopathy", Society for Neuroscience Abstracts, 2000.

57 Anderson N. E., Kiehl K. A., "The psychopath magnetized: insights from brain imaging", Trends in Cognitive Sciences, 16, 2012, pp. 52-60.

${ }^{58}$ Hare R. D., Hare Psychopathy Checklist-Revised manual. Multy-Health Systems, Toronto, 1991. sentimentali brevi; 16) Delinquenza giovanile o infantile; 17) Versatilità criminale.

Hare definisce questi soggetti come manipolatori che usano l'intimidazione attraverso la violenza per controllare gli altri e per soddisfare i propri bisogni affermando, infine, che è il loro egocentrismo a renderli particolarmente pericolosi ${ }^{59}$.

\section{Neurocriminologia del serial killer.}

Negli ultimi anni, nelle ricerche su comportamenti violenti si sono prese in considerazione le influenze biologiche e la loro interazione con l'ambiente ${ }^{60}$. A seguito di ciò si è sviluppata la disciplina denominata neurocriminologia. In alcuni casi, l'indagine genetica in combinazione con le tecniche di analisi anatomica del funzionamento cerebrale potrebbe contribuire alla risoluzione dei casi di omicidi seriali61. Uno studio che rafforza le ipotesi della neurocriminologia, ovvero la relazione tra genetica e crimini, ha analizzato 43.243 figli adottati e 1.258 .826 figli naturali confermando che il figlio biologico di un soggetto condannato per un crimine violento ha maggiori probabilità di commettere a sua volta un crimine. Complessivamente, i risultati dei vari studi sulla genetica comportamentale documentano l'esistenza di un'ereditarietà del comportamento aggressivo in bambini,

59 Hare R. D., "Psychopathy and antisocial personality disorder: A case of diagnostic confusion", Psychiatric Times, 132, 1997.

${ }^{60}$ Moya-Albiol L., Psicobiologia de la violencia, Piramide, Madrid, 2010.

${ }^{61}$ Raine A., Dogra T.D., Leenaars A.A., Yadav B., Bhera C., Lalwani S., Leenars L., "Identify of victims from fragmented and decomposed remmants by DNA profiling in a case of serial killings", Journal of Medicine Science of Law, 50, 2010, pp. 220-223. 
adolescenti e adulti. Tali risultati mostrano che vi è un contributo genetico alla criminalità.

In Italia al momento, la posizione della neurocriminologia, sia in ambito accademico sia peritale forense, è molto critica. I criminologi si sono opposti per molti anni alla tesi che sostiene che gli individui abbiano una predisposizione genetica nei confronti della violenza, le cui basi teoriche nascono dalla discussa teoria di Lombroso.

Le ammino ossidasi o monoammino ossidasi (MAO) sono enzimi legati al cofattore-FAD, appartenenti alla classe degli ossidoreuttasi. I geni che codificano per le MAO sono situati sul braccio corto del cromosoma X. Quando il gene risulta meno attivo, le persone che hanno avuto un'infanzia difficile hanno una probabilità dieci volte maggiore di essere condannate per violenze o per comportamenti aggressivi. Conosciuto anche come "warrior gene", la cui funzione è di regolare la serotonina nel cervello e un'alterazione della variante genetica MAO-A, sarebbe presente negli omicidi seriali62. Tale ipotesi, tuttavia, per ora non è confermata da sufficienti prove sperimentali.

A differenza degli esseri umani, i rettili non sono equipaggiati con la parte del cervello responsabile della memoria, della socializzazione e delle emozioni. Pertanto, dicendo che un serial killer ucciderebbe a "sangue freddo", si potrebbe fare riferimento al fatto che la parte del suo cervello che regola le emozioni e la socializzazione non funziona correttamente. In

\footnotetext{
${ }^{62}$ Gorodetsky E., Bevilacqua L., Carli V., Sarchione M., Roy A., Goldman D., Enoch M. A., "The interactive effect of MAOA-LPR genotype and childhood physical neglect on aggressive behaviors in Italian male prisoners", Genes Brain Behavior, 13, 2014, pp. 543-549.
}

tal senso, egli si comporterebbe come un rettile che uccide senza provare alcun rimorso ${ }^{63}$. Alcuni recenti studi neuroscientifici hanno stabilito che tale anomalia si tradurrebbe in un aumento della probabilità del verificarsi di un certo numero di comportamenti quali: meno controllo sul sistema limbico che genera emozioni primordiali come la rabbia, una maggiore dipendenza psicologica dal rischio, una riduzione dell'autocontrollo, una scarsa capacità di risolvere problemi e un'assenza di empatia ${ }^{64}$.

Le principali aree cerebrali correlate all'aggressività sono: l'amigdala, l'ippocampo e le strutture tegmentali. L'amigdala è una struttura che appartiene al sistema limbico, il quale interviene nei processi della regolazione dei comportamenti, delle motivazioni, delle emozioni, dell'aggressività, dell'alimentazione, dell'apprendimento, della memoria e del comportamento sessuale. Uno studio condotto da Yang e collaboratori ${ }^{65}$ osservò, in psicopatici criminali, utilizzando la risonanza magnetica per immagini (MRI), una riduzione del 22,3\% della materia grigia prefrontale. Un esempio significativo è quello del caso di Antonio Bustamante, un assassino seriale di origini messicane, poi emigrato negli Stati Uniti, che è stato assolto dalla pena di morte in quanto la difesa ha mostrato le immagini relative al suo

63 Woodworth M., Porter S., "In cold blood: Characteristics of criminal homicides as a function of psychopathy", Journal of Abnormal Psychology, 111, 2002, pp. 436-445.

64 Raine A., Moffitt T.E., Caspi A., Loeber R., Stouthhamer-Loeber M., Lynam D., "Neurocognitive impairments in boys on the life-course persistent antisocial path", Journal of Abnormal Psychology, 114, 2005, pp. 38-49.

65 Yang Y., Raine A., Lencz T., Bihrle S., LaCasse L., Colletti P., "Volume reduction in prefrontal gray matter in unsuccessful criminal psychopaths", Biological Psychiatry, 57, 2005, pp. 1103-1108. 
funzionamento cerebrale ${ }^{66}$. Un altro caso è quello di Erbert Weinsten in quanto, attraverso la PET e la MRI, è stato messo in evidenza che gli mancava una parte della corteccia prefrontale del cervello, motivo per cui la pena inflitta fu notevolmente ridotta ${ }^{67}$.

Raine fu il primo studioso ad applicare tecniche di imaging cerebrale sui criminali. Attraverso la PET è stato dimostrato come un deficit del funzionamento della corteccia prefrontale fosse presente nei serial killer. Egli ha effettuato la PET su 41 assassini condannati e ha confrontato i risultati con un gruppo di controllo formato da soggetti di età e profilo simile. Questo studio ha mostrato l'attività metabolica in diverse parti del cervello: i cervelli degli assassini mostravano una significativa riduzione nello sviluppo della corteccia prefrontale rispetto al gruppo di controllo ${ }^{68}$. Nonostante alcune evidenze scientifiche rilevino, quindi, dei deficit a livello cerebrale nei serial killer, questo rimane un campo di ricerca che deve essere maggiormente esplorato.

Gli studi sulla popolazione generale indicano che un aumento dell'attività serotoninergica riduce l'impulsività, mentre una sua diminuzione aumenta la frequenza e l'intensità di reazioni aggressive e antisociali. Gli studi genetici indicano che il gene del recettore 5-HT1B è in relazione alla condotta impulsiva e aggressiva in soggetti con disturbo di personalità antisociale e in persone alcoliste con esplosioni intermittenti

\footnotetext{
${ }^{66}$ Raine A., The Anatomy of Violence: the biological roots of crime, Vintage books, New York, 2014.

${ }^{67}$ Rosen J., "The brain on the stand", The New York Times, 11 March 2007.

${ }^{68}$ Raine A., Sanmartin J., Violencia y Psicopatia, Ariel, Barcelona, 2006.
}

di violenza ${ }^{69}$. Allo stato attuale, nella creazione di un profilo criminologico di un omicida seriale, questo parametro non può essere preso in considerazione in quanto non sono stati ancora effettuati studi ad hoc.

In generale si ritiene che la dopamina, l'adrenalina e la noradrenalina parteciperebbero come attivatori di comportamenti aggressivi. Gli effetti più evidenti, che tuttavia non sono stati dimostrati su assassini seriali, dimostrano che una leggera attivazione del sistema noradrenergico centrale stimolerebbe atteggiamenti aggressivi. La noradrenalina influisce sulla condotta aggressiva a tre livelli differenti: endocrino, autonomo e centrale. Allo stato attuale della ricerca non è possibile stabilire una correlazione tra le due sostanze in oggetto e i comportamenti criminali di omicidi seriali.

Un danno ipotalamico può produrre un comportamento violento e distruttivo e in taluni casi può provocare l'incapacità di un individuo di separare l'eccitazione sessuale dalla violenza.

Il ruolo della corteccia prefrontale è direttamente collegato al comportamento sociale quale la violenza e l'aggressività. Se questo sistema è danneggiato, un individuo può non avere la capacità di reagire in modo adeguato alle aspettative sociali. Se il lobo temporale è danneggiato, un individuo può avere amnesia, epilessia e riduzione della soglia a risposte violente, elemento che potrebbe essere considerato in fase giudiziaria. Il cervello di alcuni criminali presenterebbe una riduzione dello sviluppo della corteccia prefrontale e, di

${ }^{69}$ Lappalainen J., Long J. C., Eggert M., Ozaki N., Robin R.W., Brown G.L., "Linkage of antisocial alcoholism to the serotonin 5-HT1B receptor gene in 2 populations", Archives of General Psychiatry, 55, 11, 1998, pp. 989-994. 
conseguenza, della funzione esecutiva del cervello ${ }^{70}$.

Nel 2000 una relazione effettuata da Richard Davidson dell'Università del Wisconsin riporta come, attraverso le scansioni cerebrali, le immagini del cervello di coloro che erano stati condannati per un omicidio, e che soffrivano di disturbi aggressivi o antisociali, mostravano un'attività cerebrale distinta rispetto a coloro che erano considerati normali. Davidson e colleghi hanno scoperto che l'attività cerebrale, nella corteccia frontale orbitale e nella corteccia cingolata anteriore, era inferiore alla norma o addirittura inesistente ${ }^{71}$.

Circa il 70\% di coloro che hanno subito un grave trauma cranico svilupperebbero tendenze aggressive. Ciò non significa che tutti i soggetti con una lesione cerebrale diventeranno omicidi seriali.

Le principali patologie nelle quali una componente surrogata è risultata essere la violenza e/o l'aggressività e in alcuni casi l'omicidio sono: l'alzheimer, l'epilessia, il danno cerebrale sopravvenuto (incidenti cerebrovascolari, traumatismi cranioencefalici e alcuni tipi di tumori) e la demenza frontotemporale ${ }^{72}$. Alcuni ricercatori sostengono che esiste una relazione tra gli effetti che portano

\footnotetext{
${ }^{70}$ Kihel K.A., Bates A.T., Laurens K.R., Hare R.D., Liddle P.F., "Brain Potentials Implicate Temporal Lobe Abdnormalities in Criminal Psychopaths", Journal of Abnormal Psychology, 115(3), 2006.

71 Davidson R. J., "Affective neuroscience and psychophysiology: Toward a synthesis", Psychophysiology, 40, 2003, pp. 655-665.

72 Martinez Ortiz M., Moya Albiol L., Blasco Ros C., Modulo IV, Diploma de Neurocriminologia, Universidad de Valencia, 2014.
}

un assassino seriale a commettere gli omicidi e gli effetti indotti dalle crisi epilettiche. ${ }^{73}$

Non abbiamo riscontrato evidenza scientifica reltivamente alla presenza di tumori cerebrali tra i serial killer. Lesioni cerebrali non specifiche sono state osservate in alcuni assassini seriali tra $i$ quali Leonard Lake, David Berkowitz, Kennet Bianchi, John Gacy e Carl Panzram. Anche questa, pertanto, rimane una variabile da verificare scientificamente.

La demenza frontotemporale si caratterizza per alterazioni della condotta. Nonostante induca cambiamenti radicali nella personalità, quali ad esempio impulsività e condotte antisociali, questo tipo di demenza non può essere considerato un elemento di rilievo nella costruzione di un profilo criminologico in quanto sono assenti studi su comportamenti umani violenti.

Sono stati osservati casi di omicidi seriali commessi da soggetti con criteri diagnostici dello spettro autistico ed in particolare del disturbo di Asperger ${ }^{74}$. Secondo una ricerca svolta da studiosi dell'Università di Glasgow, il 10\% degli assassini seriali mondiali mostrerebbe caratteristiche della Sindrome di Asperger e circa la stessa percentuale presenterebbe un trauma cranico, mentre secondo una ricerca condotta da Allely e collaboratori la percentuale salirebbe al $28 \%$ per l'autismo e al $21 \%$ per un indefinito trauma cranico ${ }^{75}$.

73 Simkin M.V., Roychowdhury V.P., "Stochastic modelling of a serial killer", Journal of Theorical Biology, 355, 2014, pp. 11-16.

${ }^{74}$ Silva J.A., Leong G.B., Ferrari M.M., “A neuropsychiatric developmental moder of serial homicidal behavior", Behavioral Science and the Law, 22, 2004, pp. 787-799.

75 Allely C., Minnis H., Thompson L., Wilson P., Gillberg C., "Neurodevelopmental and psychosocial risk factors in serial killers and mass murderers. 


\section{Conclusioni.}

Gli studi sull'assassino seriale sono, a tutt'oggi, centrati su obiettivi pragmatici aventi la finalità di conoscerli in maniera descrittiva per aumentare la probabilità di catturarli. La scarsa letteratura scientifica in merito si è quindi dedicata prevalentemente a classificarli piuttosto che a studiarli.

Allo stato attuale si è quasi del tutto concordi sulla criminodinamica e la criminogenesi delle uccisioni seriali, anche se tuttavia permane una certa difficoltà ad uniformare le definizioni. L'omicida seriale "normale" non soffrirebbe quindi né di un disturbo mentale o né di un disturbo della personalità.

Da un punto di vista neurobiologico il fenomeno si è studiato con l'intenzione di scoprire eventuali alterazioni delle attività cerebrali. La psicologia ha seguito la via psicopatologica cercando di identificare un disturbo mentale che la spiegasse. Il punto di vista criminologico cerca di contestualizzare il fenomeno dal punto di vista legale, ovvero soffermandosi sulla questione dell'imputabilità dell'omicida seriale, cercando di capire se egli è malato o semplicemente se è una persona malvagia. Emerge quindi l'importanza di un approccio integrato in cui la neurocriminologia potrebbe contribuire a rispondere a domande del tipo: "La società può creare luoghi appositi di reinserimento di queste persone?", "Si può prevenire tale fenomeno?".

Lo studio degli omicidi seriali presenta vari problemi. Il primo è che il campione oggetto di studio difficilmente può essere rappresentativo e il secondo, correlato al primo, riguarda il fatto che i pochi dati ottenuti spesso si riferiscono a fonti non scientifiche e approssimative.

Come abbiamo visto, molti parametri oggetto di studio nei comportamenti aggressivi e/o violenti non sono sufficientemente valutati o parzialmente osservati negli omicidi seriali. Tra i fattori che ipotizziamo possano essere significativi nella costruzione di un profilo neurocriminologico, che comunque andrebbero maggiormente studiati, sono la presenza del warrior gene e di anomalie nella corteccia prefrontale. In base alle conoscenze attuali, il profilo criminologico dell'omicida seriale non può essere ricondotto unicamente a variabili neurocriminologiche.

Attualmente gli elementi dei quali dispone la neurocriminologia non sono di per sé sufficienti per definire un profilo neurocriminologico di un omicida seriale. Emergerebbe quindi l'importanza di un approccio che dovrebbe coinvolgere più discipline come la criminologia, la psichiatria forense, la psicologia giuridica e la neurocriminologia. Tali discipline, organizzate in un modello multidisciplanare, potrebbero contribuire alle attività criminologiche quali, ad esempio, la prevenzione di condotte criminali, consulenze tecniche psicologiche in ambito penale, attività peritale di profili criminologici e collaborazione con le forze dell'ordine nella risoluzione dei casi. 


\begin{tabular}{|c|c|}
\hline Serial killer organizzato & Serial killer disorganizzato \\
\hline QI nella media o al di sopra della media & QI sotto la media \\
\hline Socialmente competente & Socialmente inadeguato \\
\hline Sessualmente competente & Sessualmente incompetente \\
\hline Disciplina inconsistente durante l'infanzia & Disciplina rigida durante l'infanzia \\
\hline Umore controllato durante la commissione dei crimini & Ansia durante la commissione dei crimini \\
\hline Abuso di alcol durante la commissione dei crimini & Minimo o assente uso di alcol durante la commissione \\
& dei crimini \\
\hline Stress situazionale precipitante & Stress situazionale minimo \\
\hline Mobilità con l'autovettura & \\
\hline Segue le notizie sui delitti & Scarso o assente interesse per le notizie sui delitti \\
\hline
\end{tabular}

Tabella n. 1: Classificazione F.B.I. - Serial killer organizzato e disorganizzato

\begin{tabular}{|l|l|}
\hline Tratto psicopatico & Disfunzione neuorchimica \\
\hline Violenza & $\begin{array}{l}\text { 5-HIAA (+) Testosterone (+) } \\
\text { Serotonina }(-)\end{array}$ \\
\hline Impulsività & COMT (+) \\
\hline Insensibilità & Dopamina (-) \\
\hline Assenza di empatia & Testosterone (+) \\
\hline Resistenza allo stresso & Cortisolo (+) Testosterone (+) \\
\hline Resistenza alla punizione & Dopamina (-) \\
\hline
\end{tabular}

Tabella n. 2: Neurochimica e psicopatia

\section{Bibliografia.}

- Alison L., Bennell C., Mokros A., Ormerod, D., "The personality paradox in offender profiling: A theoretical review of the processes involved in deriving background characteristics from crime scene actions", Psychology, Public Policy and Law, 8, 2002, pp. 115-135.

- Allely C., Minnis H., Thompson L., Wilson P., Gillberg C., "Neurodevelopmental and psychosocial risk factors in serial killers and mass murderers. Aggression and Violent Behavior", in American Psychiatric Association, DSM-V, 2014.

- Anderson N. E., Kiehl K. A., "The psychopath magnetized: insights from brain imaging", Trends in Cognitive Sciences, 16, 2012, pp. 52-60.

- Balloni A., Bisi R., Monti C. (a cura di), Soda caustica, allume di rocca e pece greca. Il caso Cianciulli, Minerva Editore, Bologna, 2010.

- Bandura A., Aggression: A social learning analysis, Prentice-Hall, Englewood Cliffs, NJ, 1973.

- Baron R. A., Byrne D., Social psychology (10th edition), Allyn \& Bacon, Boston, 2003.

- Blair R. J., "Neurobiological basis for psychopathy", British Journal of Psychiatry, 182, 2003, pp. 5-7.
- Bisi R. (a cura di), Psicodiagnostica e storie di vita in criminologia. Un'analisi attraverso l'omicidio, FrancoAngeli, Milano, 2004.

- Canter D. V., Alison L. J., Alison E., Wentink N., "The organized/disorganized typology of serial murder: Myth or model?", Psychology, Public Policy and Law, 10, 2004, pp. 293-320.

- Davidson R. J., "Affective neuroscience and psychophysiology: Toward a synthesis", Psychophysiology, 40, 2003, pp. 655-665.

- Dietz P. E., "Mass, serial and sensational homicides", Bulletin of the New York Academic of Medicine, 62(5), 1986, pp. 477-491.

- Dogra T. D., Leenaars A. A., Chadha R. K., Manju M., Laiwani S., Sood M., Lester D., Raine E., Behera C., "A psychological profile of a serial killer: a case report", Omega, 65, 2012, pp. 299-316.

- Douglas J. E., Burgess A.W., Burgess A.G., Ressler R.K., Crime classification manual: $A$ standard system for investigating and classiffing violent crimes, Simon and Schuster, New York, 1992.

- Edelstein A., "Re-thinking typologies of multiple murders: the missing category of serial-mass murder and its theoretical and practical implications", Journal of International Mental Health, 16, 2014, pp. 350-353. 
- Egger S.A., The killer among us: an examination of serial murder and its investigation, Prentice Hall, Upper Saddle River, NJ, 1998.

- Eysenck H.J., Eysenck S.B.G., Psychoticism as a dimension of personality, Hodder and Stoughton, London, 1976.

- Fisher K., Fisher R., Common characteristics of a serial killer, 2003. Retrieved August 8, 2014 from http://www.carpenoctem.tv

- Gacono C. B., Hutton H. E., "Multiple murder: a review", Journal of British Criminology, Delinquency and Deviant Social Behavior, 34, 1, 1994, pp. 1-14.

- Gao Y., Raine A., Venables P. H., Dawson M. E., Mednick S. A., "Association of poor childhood fear conditioning and adult crime", American Joumal of Psychiatry, 167, 2010, pp. 56-60.

- Gorodetsky E., Bevilacqua L., Carli V., Sarchione M., Roy A., Goldman D., Enoch M. A., "The interactive effect of MAOALPR genotype and childhood physical neglect on aggressive behaviors in Italian male prisoners", Genes Brain Behavior, 13, 2014, pp. 543-549.

- Groth A. N., Burgess A.W., Holmstrom L.L., "Rape: Power, anger and sexuality", American Journal of Psychiatry, 134(11), 1977, pp. 1239-1243.

- Harbort S,. Mokros M., "Serial Murderers in Germany from 1945 to 1995. A descriptive study", Homicide Studies, vol. 5, No. 4, 2001, pp. 311-334.

- Hare R. D., Hare Psychopathy Checklist-Revised manual. Multy-Health Systems, Toronto, 1991.

- Hare R. D., "Psychopathy and antisocial personality disorder: A case of diagnostic confusion", Psychiatric Times, 132, 1997.

- Hickey E. W., "Serial murderers and theirs victims", Wadsworth Publishing Company, Belmont, 1997.

- Holmes R., Holmes S., "Understanding mass murder: a starting point", Federal Probation, 49, 1992, pp. 29-34.

- Holmes R.M., DeBerger J., Serial Murder, Sage, Newbury Park CA, 1988.

- Kiehl K.A., Smith A.M., Hare R.D., "Limbic abmormalities in affective processing by criminal psychopaths as revealed by functional magnetic resonance imaging", Biological Psychiatry, 50, 2001, pp. 677-684.

- Kihel K.A., Bates A.T., Laurens K.R., Hare R.D., Liddle P.F., "Brain Potentials Implicate Temporal Lobe Abdnormalities in
Criminal Psychopaths", Journal of Abnormal Psychology, 115(3), 2006.

- Kraemer G.W., Lord W.D., Heilburn K., "Comparing single and serial homicide offenses", Behavioral Science of Law, 22, 2004, pp. 325-343.

- Lahey B.B., Loeber R., Burke J.D., Applegate B, "Predicting future antisocial personality disorder in males from a clinical assessment in childhood", Journal of Counseling and Clinical Psychology, 73, 2005, pp. 389-399.

- Lappalainen J., Long J. C., Eggert M., Ozaki N., Robin R.W., Brown G.L., "Linkage of antisocial alcoholism to the serotonin 5HT1B receptor gene in 2 populations", Archives of General Psychiatry, 55, 11, 1998, pp. 989-994.

- Loeber R., Pardini D., Homish L., Wei E. H., Crawford A. M., Farrington D.P., "The prediction of violence and homicide in young men", Journal of Counseling and Clinical Psychology, 73, 2005, pp. 1074-1088.

- Martinez Ortiz M., Moya Albiol L., Blasco Ros C., Modulo IV, Diploma de Neurocriminologia, Universidad de Valencia, 2014.

- Mastronardi V., Manuale per operatori criminologici e psicopatologi forensi, Giuffrè, Milano, 2012.

- Mitchell H., Aamodt M.G., "The incidence of child abuse in serial killers", Journal of Police and Criminal Psychology, 20, 2005, pp. 4047.

- Moffitt T.E., "The new look of behavioral genetics on developmental psychology: Gene-environment interplay in antisocial behaviors", Psychological Bullettin, 131, 2005, pp. 533-535.

- Montaldo C., Characteristics of a Psychopatbic Personality, 2007. Retrieved January 10, 2015 from http://www.crime.about.com

- Moya-Albiol L., Psicobiologia de la violencia, Piramide, Madrid, 2010.

- Moya-Albiol L., La empatía. Entenderla para entender a los demás, Plataforma, Barcelona, 2014.

- Myers W.C., "Serial murder by children and adolescents", Behavioral Science of Law, 22, 2004, pp. 357-374.

- Newton M., The Encyclopedia of serial killers, 2 ed., Checkmark Books, New York, 2006.

- Ostrosky-Solis F., Velez-Garcia A., SantanaVargas D., Perez M., Ardilla A., "A middle- 
aged female serial killer", Journal of Forensic Science, 53, 2008, pp. 1223-1230.

- Petherick W.A., Serial crime: Theoretical and Practical issues in behavioral profiling, Elsevier, California, 2006.

- Raine A., Sanmartin J., Violencia y Psicopatia, Ariel, Barcelona, 2006.

- Raine A., Dogra T.D., Leenaars A.A., Yadav B., Bhera C., Lalwani S., Leenars L., "Identify of victims from fragmented and decomposed remmants by DNA profiling in a case of serial killings", Journal of Medicine Science of Law, 50, 2010, pp. 220-223.

- $\quad$ Raine A., Moffitt T.E., Caspi A., Loeber R., Stouthhamer-Loeber M., Lynam D., "Neurocognitive impairments in boys on the life-course persistent antisocial path", Journal of Abnormal Psychology, 114, 2005, pp. 38-49.

- Rappaport R., "The serial and muss murder: patterns, differentiation, pathology", American Journal of Forensic Psychiatry, 9, 1988, pp. 39-48.

- Ressler R. K., Burgess A.W., Douglas J.E., Sexual Homicide: patterns and motives, Iosington, New York, 1998.

- Rice M.E., "Violent offender research and implications for the criminal justice system", American Psychologist, 52, 1997, pp. 414-423.

- Rosen J., "The brain on the stand", The New York Times, 11 March 2007.

- Salekin R.T., Leistico A.M., Neumann C.S., DiCicco T.M., Duros R.L., "Psychopathy and comorbidity in a young offender sample: Taking a closer look at psychopathy's potential importance over disruptive behavior disorders", Journal of Abnormal Psychology, 113, 2004, pp. 416-427.

- Salfati G., Canter D., "Differentiating Stranger Murders: Profiling Offender Characteristics from Behavioral Styles", Behavioral Sciences and the Law, 17, 1999, pp. 391-406.

- Sears D.J., To kill again: The motivationand development of serial murder, Scholarly Resources, Inc., Wilmington, DE, 1991.

- Silva J. A., Ferrari M.M., Leong G.B., "The case of Jeffrey Dahmer: Sexual serial homicide from a neuropsychiatric developmental perspective", Journal of Forensic Sciences, 47, 2002, pp. 1-13.

- Silva J.A., Leong G.B., Ferrari M.M., "A neuropsychiatric developmental moder of serial homicidal behavior", Behavioral Science and the Law, 22, 2004, pp. 787-799.
- Simkin M.V., Roychowdhury V.P., "Stochastic modelling of a serial killer", Journal of Theorical Biology, 355, 2014, pp. 1116.

- Sycamnias E., Evaluating a Psychological Profile of a Serial Killer, 2007. Da: http://www.crime.about.com

- Taylor J. Iacono W.G., McGue M., "Evidence for a genetic etiology of earlyonset delinquency", Journal of Abnormal Psychology, 109, 2000, pp. 634-643.

- Turvey B., Criminal profiling. An introduction to behavioral evidence analysis, Elservier, California, 2008.

- van Winkel R., van Nierop M., MyinGermeys I., van Os J., "Childhood trauma as a cause of psychosis: linking genes, psychology and biology", Can J Psychiatry, 58, 2013, pp. 44-51.

- Warren J., Hazelwood R.R., Dietz P.E., "The sexually sadistic serial killer", Joumal of Forensic Science, 41, 1996, pp. 970-974.

- White J.H., Lester D., Gentile M., Rosenbleeth J., "The utilization of forensic science and criminal profiling for capturing serial killers", Journal of Forensic Science International, 209, 2011, pp. 160-165.

- Wilson W., Hilton T., "Modus operandi of female serial killers", Psychology Rep., 82, 1998, pp. 495-498.

- Wolf G., "A kidney from hell? A nephrological view of the Whitechapel murders in 1888", Nephrology Dialysis Transplantation, 23, 2008, pp. 3343-3349.

- Woodworth M., Porter S., "In cold blood: Characteristics of criminal homicides as a function of psychopathy", Journal of Abnormal Psychology, 111, 2002, pp. 436-445.

- Yang Y., Raine A., Lencz T., Bihrle S., LaCasse L., Colletti P., "Volume reduction in prefrontal gray matter in unsuccessful criminal psychopaths", Biological Psycbiatry, 57, 2005, pp. 1103-1108.

- Yorker B.C., Kizer K.W., Lampe P., Forrest A.R., Lannan J.M., Russell D.A., "Serial muder by healthcare professionals", Journal of Protocol Manage, 24, 2008, pp. 63-77. 